\title{
Total Retrograde Conduction Block by Higher Dose Bisoprolol in Permanent Junctional Re-Entry Tachycardia and Severe Dilated Tachymyopathy
}

\author{
Dr. Stefan Peters \\ Chair Cardiology, St.Elisabeth Hospital gGmbH Salzgitter, Germany \\ H.u.S.Peters@t-online.de
}

A meanwhile 45-year male patient suffered from initial permanent junctional reentrant tachycardia with a prolonged RP > PR interval and a severe form of dilated cardiomyopathy reported in advance in two publications $(1,2)$.

The patient was sent to an electrophysiological study where a retrograde conduction block in the atrioventricular node was documented under high dose bisoprolol (7.5mg per day). A dual conduction physiology in the atrioventricular node was not present. With the exclusion of permanent junctional re-entrant tachycardia the patient was sent home.

At the beginning of 2018 was sent to a control examination (ECG, echocardiography). The patient had no complaints.

ECG showed sinus rhythm at a heart rate of $65 / \mathrm{min}$, positive P waves in leads II, III and aVF, no signs of PJRT under continuous medication with entresto (100mg td), high dose bisoprolol (7,5mg td) and spironolacton (25mg).

Echocardiography revealed normal dimension and an increase in left ventricular function with an ejection fraction of 52\%. Slight mitral insufficiency was seen.

In conclusion, the patient was sent to electrophysiological laboratory to do catheter ablation of permanent junctional re-entrant tachycardia. In the diagnostic approach there was no dual conduction physiology due to complete conduction block in the atrioventricular node.

High dose bisoprolol has strict beta-blocking properties and functional refractory periods of the AV node (3). Under intravenous administration bisoprolol has depressant effects mainly of atrial function and the nodal conduction (4).

In this special patient orally administrated bisoprolol in higher dose leads to a complete retrograde conduction block of the AV node. Ablation of PJRT was not necessary at all. The patient had no complaints, the medication was continued in the follow-up.

In children at lower age amiodarone, flecainide, maybe in combination with digitalis, is used to treat PRJT (5). Definite cure is only possible by catheter ablation in advanced age. In adult patients PJRT presented with longer duration of symptoms, more frequently tachycardia-induced cardiomyopathy and a slower tachycardia rate (6).

Antiarrhythmic medication - in this a case high dose beta blocking agents - can provide no recurrence of permanent junctional re-entry tachycardia thus solving the problem of tachymyopathy 
High Total Retrograde Conduction Block by Higher Dose Bisoprolol in Permanent Junctional Re-Entry Tachycardia and Severe Dilated Tachymyopathy

\section{REFERENCES}

1. Peters S. Tachycardiomyopathy: A case of dilated cardiomyopathy due to permanent junctional reentrant tachycardia. Int J Cardiol 2016: 207: 233

2. Peters S. Recurrence of permanent junctional re-entry tachycardia: indication for ablation of the junctional pathway. Int J Cardiol 2016; 220: 653

3. Procelemer A, Gradnik R, Savonitto S, Feruglio GA. Electrophysiological effects of bisoprolol. Eur Heart J 1987, Supll M: 81 - 5

4. Clementy J, Samoyeau R, Coste P, Bricaud H. Study of the electrophysiological properties of intravenous bisoprolol in patients with and without coronary artery disease by programmed stimulation. J Cardiovasc Pharmacol 1990; Suppl 5: S 169 - 74

5. Lindinger A, Heisel A, von Bernuth G, et al. Permanent junctional re-entry tachycardia. Eur Heart J 1998; 19: $936-41$

6. Meiltz, A,Weber R, Halimi F, Defaye P, Boveda S, Tavornier R, et al. Permanent form of junctional reciprocating tachycardia in adults: peculiar features and results of radiofrequency catheter ablation. Europace 2006; 8: 21 - 6

Citation: S Peters. "Total Retrograde Conduction Block by Higher Dose Bisoprolol in Permanent Junctional Re-Entry Tachycardia and Severe Dilated Tachymyopathy". American Research Journal of Cardiovascular Diseases. 2018, 2(1); pp: 1-2.

Copyright (C2018 S Peters. This is an open access article distributed under the Creative Commons Attribution License, which permits unrestricted use, distribution, and reproduction in any medium, provided the original work is properly cited. 